\title{
Influence of Site Differences between Urban and Rural American and Central European Opioid-Dependent Pregnant Women and Neonatal Outcome Characteristics
}

\author{
Andjela Baewert ${ }^{a}$ Reinhold Jagsch ${ }^{b}$ Bernadette Winklbaur ${ }^{a}$ Gerda Kaiser $^{a}$ \\ Kenneth Thau ${ }^{\mathrm{a}}$ Annemarie Unger ${ }^{\mathrm{a}}$ Constantin Aschauer ${ }^{\mathrm{a}}$ Manfred Weninger ${ }^{\mathrm{c}}$ \\ Verena Metz ${ }^{a}$ \\ ${ }^{a}$ Department of Psychiatry and Psychotherapy, Medical University Vienna, ${ }^{b}$ Faculty of Psychology, \\ University of Vienna, and ' Department of Neonatology, Medical University Vienna, Vienna, Austria
}

\section{Key Words \\ Opioid dependence $\cdot$ Pregnancy $\cdot$ Methadone $\cdot$ \\ Buprenorphine $\cdot$ Maternal and neonatal outcome $\cdot$ \\ Regional disparities}

\begin{abstract}
Background: Multi-center trials enable the recruitment of larger study samples, although results might be influenced by site-specific factors. Methods: Site differences of a multicenter prospective double-blind, double-dummy randomized controlled trial (7 centers: Central Europe (Vienna)/USA (3 urban/3 rural centers)) comparing safety and efficacy of methadone and buprenorphine in pregnant opioid-dependent women and their neonates. Results: Urban US women had the highest rate of concomitant opioid $(p=0.050)$ and cocaine consumption $(p=0.003)$, the highest dropout rate $(p=0.001)$, and received the lowest voucher sums $(p=0.001)$. Viennese neonates had significantly higher Apgar scores 1 min $(p=0.001)$ and 5 min after birth $(p<0.001)$ and were more often born by cesarean section $(p=0.024)$. Rural US newborns had a significantly shorter neonatal abstinence syndrome treatment duration compared to Viennese and urban US sites $(p=0.006)$, in addition to other site-specific differences, suggesting a more severely affected group of
\end{abstract}

women in the urban US sites. Conclusion: This clinical trial represents a role model for pharmacological treatment in this unique sample of pregnant women and demonstrates the clinical importance of considering site-specific factors in research and clinical practice.

Copyright $\odot 2012$ S. Karger AG, Basel

\section{Introduction}

According to estimates of the United Nations Office of Drugs and Crime (UNODC), between 12.8 and 21.9 million people worldwide abused opioids in 2008 , with the prevalence ranging from 0.3 to $0.5 \%$ among the population aged 15-64 years [1]. As one third of the patients are women of childbearing age, drug abuse during pregnancy represents a major public health concern, also particularly because of associated adverse neonatal outcomes entailing intensive medical care at high costs [2].

Substance use disorders and associated behaviors and consequences, ranging from self-neglect, poor nutrition, high-risk sexual behavior to infectious diseases through intravenous drug abuse, might have harmful effects on the pregnant women and their unborn children $[3,4]$. A lack of access to treatment or problems

\section{KARGER}

Fax +4161306 1234

E-Mail karger@karger.ch

www.karger.com (c) 2012 S. Karger AG, Basel

$1022-6877 / 12 / 0183-0130 \$ 38.00 / 0$

Accessible online at:

www.karger.com/ear
Andjela Baewert

Department of Psychiatry and Psychotherapy, Medical University Vienna

Währinger Gürtel 18-20

AT-1090 Vienna (Austria)

Tel. +43 140400 3547, E-Mail andjela.baewert@meduniwien.ac.at 
with compliance frequently results in unplanned pregnancies or delayed identification and subsequently late start of antepartum care as well as gynecological/obstetrical complications such as premature delivery, stillbirth, or placental disruption [5-7]. Moreover, psychiatric comorbidities, in particular anxiety disorders and depression, are highly prevalent, frequently untreated, and further complicate treatment of this special patient population [8].

Although opioid maintenance treatment with methadone has been established as the first-line treatment for opioid-dependent pregnant women in most industrialized countries, recent publications have reported effectiveness and fetal safety of buprenorphine [9-12]. Two independent randomized controlled trials (RCTs) conducted by Jones et al. [13] and Fischer et al. [14] provided data suggesting that buprenorphine may be well suited as maintenance medication in opioid-dependent pregnant women. These two pilot trials were precursors for the larger multi-site study 'Maternal Opioid Treatment: Human Experimental Research (MOTHER)', the subject of this secondary analysis $[15,16]$. The intent of the MOTHER study was to recruit a sufficiently large sample of opioid-dependent women to generate evidence for the safe and efficient application of methadone and buprenorphine during pregnancy [9]. The main finding of the MOTHER trial refers to the safety of both medications with superior effects of buprenorphine yielding significantly shorter treatment durations for neonatal abstinence syndrome (NAS) and associated lower medication requirement and hospital stays in neonates. However, women maintained on buprenorphine were more likely to discontinue their treatment due to medication dissatisfaction, suggesting that the partial agonist might not be optimal for all subgroups of opioid-dependent pregnant women, particularly not for those with the most severe addictive disorders. Besides differences in treatment response and clinical outcome on the inter-individual level, results in the MOTHER study might also differ due to regional disparities of patient subgroups. An analysis of the screening sample of the clinical trial [17], dividing the pregnant women into three groups, namely US urban, US rural and Central European patients, revealed significant inter-group differences with regard to sociodemographic and clinical/substance use characteristics.

Therefore, the aim of this secondary analysis study is to investigate in depth whether these regional patient disparities found in the screening sample become also apparent in maternal and neonatal clinical outcome parameters. The analysis could though yield new insight into region-specific factors influencing the course of treatment and subsequently the clinical outcome by comparing a range of selected variables crucial for measuring treatment outcome in US urban, US rural and Central European MOTHER study completers.

\section{Materials and Methods}

\section{Study Participants}

Of 1,074 women screened, 175 (16.3\%) were blindly randomized to the study medication (buprenorphine/methadone), whereof 131 (12.2\% of screening sample; with 58 women maintained on buprenorphine and 73 on methadone) completed the study protocol, i.e. they stayed in multi-professional treatment and were following all required study procedures with daily visits at their study center until 28 days postpartum.

The MOTHER study participants had to have a singleton pregnancy, an age between 18 and 41 years, and no alcohol or benzodiazepine use disorder as well as no medical or any other conditions preventing them from following the stringent study protocol, such as pending legal issues or planned delivery at a hospital other than the study center. For a more detailed description of inclusion and exclusion criteria see also Stine et al. [15].

\section{Study Procedures}

The study participants were submitted to daily clinical procedures, including psychiatric, gynecological and other medical diagnostic and treatment interventions provided by a multi-disciplinary blinded study team, also taking care of the neonatal treatment. Since this is a secondary analysis and the main outcome publication [9] covers a detailed description of study procedures, they are only described very briefly as follows:

The MOTHER study protocol required daily supervised medication intake, three times weekly supervised urine drug screens, standardized ultrasound monitoring and delivery at the site hospital. The women received vouchers in an escalating pattern for study participation and provision of negative urine samples (screened for opiates, amphetamines, cocaine and benzodiazepines; THC was not included for contingency payments) as a form of behavioral reinforcement/contingency management.

After delivery, standardized postnatal procedures were applied for mothers and neonates. The children remained hospitalized for a minimum of 4 days (original protocol intended 10 days postpartum (performed at the Vienna site)), and standardized NAS rating by use of a modified Finnegan Scale, comprising 19 opioid withdrawal symptoms to determine the need for treatment [18], was conducted every $4 \mathrm{~h}$ during hospital stay by trained nursing staff, blinded for antenatal medication exposure. If a neonate was discharged from hospital before day 10 , it was expected that he/she would remain in a residential setting with the mother, where NAS monitoring by trained staff could be continued until 10 days after birth. However, NAS scores could only be collected twice daily in the frame of this setting [9].

NAS Treatment Protocol

According to the study protocol, NAS was treated with oral morphine drops (morphine hydrochloride at the Viennese site, 
morphine sulfate at the US sites). Pharmacotherapy was initiated when the NAS score was $\geq 9$, with morphine doses (in parentheses) selected according to the NAS score as follows: $0-8(0 \mathrm{mg})$, 9-12 (0.04 mg/dose), 13-16 (0.08 mg/dose), 17-20 (0.12 mg/dose), 21-24 (0.16 mg/dose), and $\geq 25$ (0.20 mg/dose) [15].

\section{Ethics}

Prior to treatment initiation, patients signed informed consent for trial participation and for analysis of data. The study protocol was approved by the ethics committee of each study site (for Vienna IRB No. 451/1998).

\section{Analysis/Statistics}

For the purpose of this analysis, the seven patient-contributing sites were divided into three groups (site clusters): the Vienna site $(\mathrm{n}=37)$, the rural US site $(\mathrm{n}=39)$ and the urban US site $(\mathrm{n}=$ 55). The Canadian study center was not included since it randomized no patients.

To provide comparable data for neonates, the daily mean NAS scores and the amount of medication administered over the whole time period of NAS treatment were analyzed (the mean total morphine dose, morphine dose/day and the duration of NAS treatment). The following birth outcome measurements were collected: Apgar score at 1 and $5 \mathrm{~min}$ (Apgar scores at $10 \mathrm{~min}$ were only available at the Viennese site), head circumference, birth weight, length and type of delivery (vaginal or cesarean), as well as length of stay in hospital.

\section{Statistical Procedures}

Statistical analysis was conducted in the form of a completer analysis using SPSS 16.0 for Windows. Pearson's $\chi^{2}$ tests were used for categorical data; in case of significance, the direction of the standardized residuals was used to help interpret the results.

One-way analysis of variance (ANOVA) was applied for analysis of continuous data to detect differences of means between the three groups of study sites. In case of significance, Bonferroni post-hoc tests were used to correct for alpha error.

Two-way analysis of variance (ANOVA) was used for neonatal outcome variables. Site and study drug served as independent variables. $p$ values are provided for the two main effects and for the interaction between site and study drug. Bonferroni post-hoc tests were used as well to correct for $\alpha$ error. In cases where preconditions were violated (inhomogeneous variances), alternative non-parametric test procedures were used (Kruskal-Wallis tests followed by Mann-Whitney tests).

For all comparisons, $\alpha$ of $p<0.05$ was considered significant - inter alia to generate new hypotheses and to get better comparability of results with the site differences paper regarding screening results of the MOTHER trial [17].

\section{Results}

\section{Maternal Characteristics}

Compared to both US sites, women in Vienna/Central Europe were significantly less educated $(\mathrm{p}<0.001)$. For all other sociodemographic characteristics significant differences were found between urban US and Viennese women, but not between rural US and Viennese participants. Urban US women were older $(\mathrm{p}<0.001)$, more often non-Caucasian $(\mathrm{p}<0.001)$, less often married ( $\mathrm{p}=$ $0.027)$, and less often employed $(p=0.011)$ than women from Vienna. No significant differences were found for rates of legal involvement between the sites. Concerning BMI, urban US women scored significantly higher than Viennese women $(\mathrm{p}=0.031)$, but not higher than women from the rural US sites (table 1).

Significant differences in screening modalities between the three site groups were revealed ( $<<0.001$ ); while screening in Vienna was conducted exclusively face to face, rural US sites mainly screened by phone (62\%), whereas about one third of the women of the urban US sites were screened by means of medical chart review. No significant differences were found for the age of first heroin use and the mean number of cigarettes smoked/day between the three sites. Women of the urban US sites had a higher percentage of polysubstance use (36\%) compared to the other two site groups $(\mathrm{p}=0.004)$, spent much more money on drugs $(\mathrm{p}<0.001)$, had a longer history of prior heroin consumption $(\mathrm{p}=0.002)$ and a higher Addiction Severity Index [19] drug composite score at time of screening $(\mathrm{p}<0.001)[17]$.

During the course of study participation until delivery, significantly higher rates of concomitant opioid $(\mathrm{p}=0.050)$ and cocaine consumption ( $\mathrm{p}=0.003$ ) were found for women at urban US sites compared to the Central European/ Vienna site, while no differences resulted for benzodiazepines between the three site clusters $(\mathrm{p}=0.328)$. Regarding positive urine toxicology results for THC, women of the rural US sites showed significantly higher rates than the two other sites clusters $(p<0.001)$ (table 2). Correspondingly, a significantly lower amount of vouchers was dispensed to urban US women compared to Viennese $(\mathrm{p}=0.003)$ and rural US $(\mathrm{p}=0.001)$ study participants.

Regarding mean doses of study drug at time of delivery, urban US women had a significantly higher mean methadone $(103.00 \mathrm{mg})$ and buprenorphine dose $(21.60 \mathrm{mg})$ than Viennese and rural US women $(\mathrm{p}<0.001)$.

The urban US site showed the highest dropout rate with 32 women during pregnancy (i.e. $37 \%$ of enrolled 87 subjects) compared to the two other site clusters ( $\mathrm{p}=$ 0.001). The Vienna and US rural sites had lower dropout rates with 10 and $17 \%$, respectively, over a mean study duration of 21 weeks until delivery, with almost equally distributed medication groups with $50 \%$ of women on methadone/buprenorphine. At the urban US sites, $64 \%$ of dropouts were medicated with buprenorphine. Almost $50 \%$ of 'early study discontinuation' (17 out of 32 ran- 
Table 1. Sociodemographic screening characteristics of study completers at the three site clusters

\begin{tabular}{|c|c|c|c|c|c|c|c|}
\hline & \multirow{2}{*}{$\begin{array}{l}\text { Urban USA } \\
(\mathrm{n}=55) \\
\text { mean } \pm \mathrm{SD}\end{array}$} & \multirow{2}{*}{$\begin{array}{l}\text { Vienna } \\
(\mathrm{n}=37) \\
\text { mean } \pm \mathrm{SD}\end{array}$} & \multirow{2}{*}{$\begin{array}{l}\text { Rural USA } \\
(\mathrm{n}=39) \\
\text { mean } \pm \mathrm{SD}\end{array}$} & \multicolumn{4}{|c|}{ Significance } \\
\hline & & & & $\mathrm{p}_{\text {site }}$ & $\mathrm{p}_{\mathrm{PH} 1}$ & $\mathrm{p}_{\mathrm{PH} 2}$ & $\mathrm{p}_{\mathrm{PH} 3}$ \\
\hline Education, years & $11.87 \pm 1.96$ & $10.11 \pm 1.71$ & $12.16 \pm 1.60$ & $<0.001$ & $<0.001$ & 1.000 & $<0.001$ \\
\hline $\mathrm{BMI}, \mathrm{kg} / \mathrm{m}^{2}$ & $26.35 \pm 5.85$ & $23.25 \pm 5.83$ & $25.82 \pm 4.99$ & 0.030 & 0.031 & 1.000 & 0.153 \\
\hline Ethnicity & & & & $<0.001^{\mathrm{a}}$ & & & \\
\hline Non-Caucasian & $15(27 \%)$ & $1(3 \%)$ & $0(0 \%)$ & & & & \\
\hline Marital status & & & & $0.027^{\mathrm{a}}$ & & & \\
\hline Unmarried & $53(96 \%)$ & $29(78 \%)$ & $33(85 \%)$ & & & & \\
\hline Married & $2(4 \%)$ & $8(22 \%)$ & $6(15 \%)$ & & & & \\
\hline Employment status & & & & $0.011^{\mathrm{a}}$ & & & \\
\hline Unemployed & $52(95 \%)$ & $30(81 \%)$ & $28(72 \%)$ & & & & \\
\hline
\end{tabular}

$\mathrm{p}_{\text {site }}=$ Probability site cluster; $\mathrm{p}_{\mathrm{PH} 1}=$ probability post-hoc-1 urban USA vs. Vienna; $\mathrm{p}_{\mathrm{PH} 2}=$ probability post-hoc-2 urban USA vs . rural USA; $\mathrm{p}_{\mathrm{PH} 3}=$ probability post-hoc-3 Vienna vs. rural USA.

a Pearson's $\chi^{2}$ test.

Table 2. Method of patient recruitment and drug use characteristics of the three site clusters

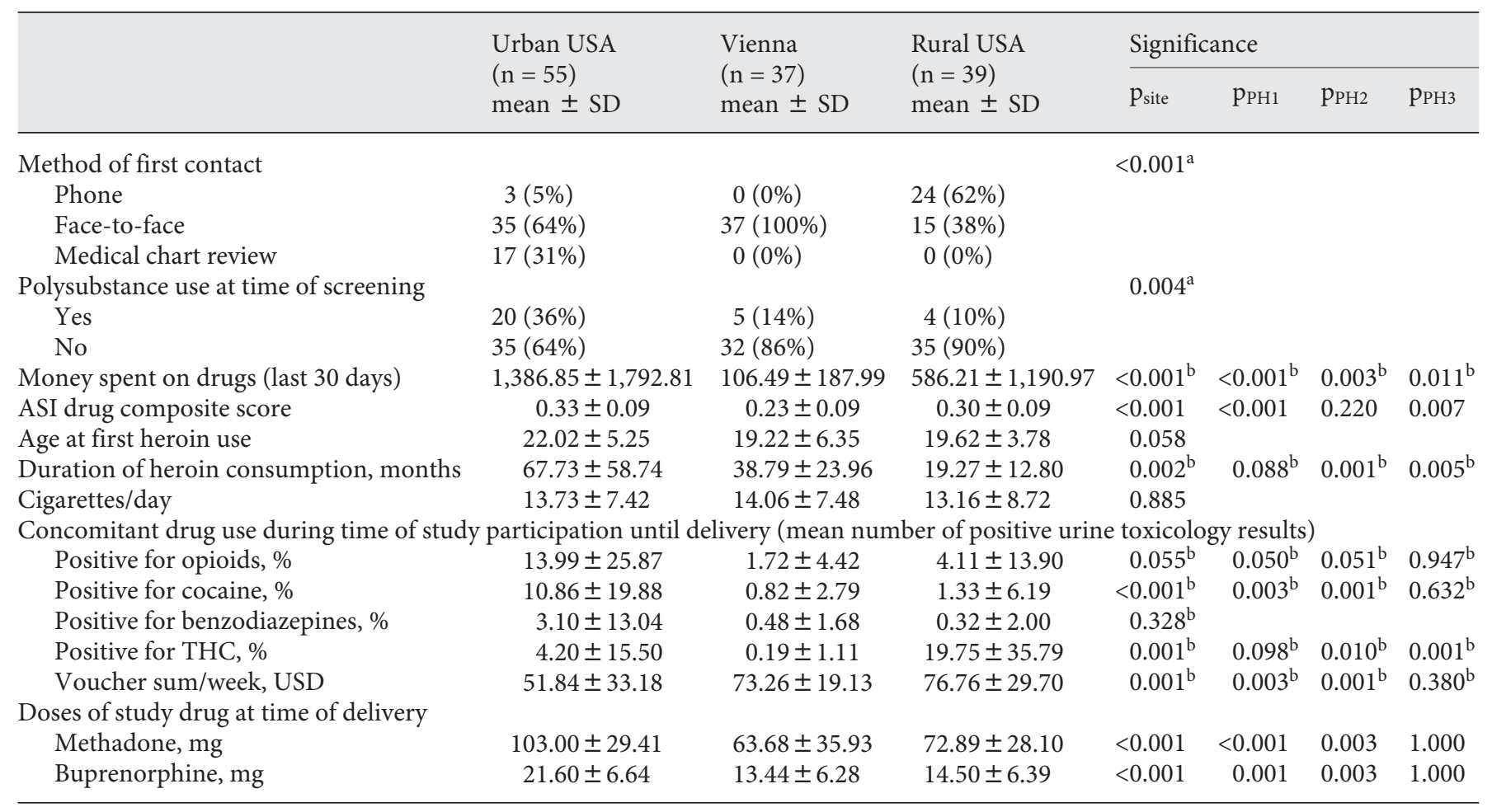

$\mathrm{p}_{\text {site }}=$ Probability site cluster; $\mathrm{p}_{\mathrm{PH} 1}=$ probability post-hoc-1 urban USA vs. Vienna; $\mathrm{p}_{\mathrm{PH} 2}=$ probability post-hoc- 2 urban USA vs. rural USA; $\mathrm{p}_{\mathrm{PH} 3}=$ probability post-hoc-3 Vienna vs. rural USA.

a Pearson's $\chi^{2}$ test. ${ }^{\mathrm{b}}$ Non-parametric tests (Kruskal-Wallis and Mann-Whitney tests). 
Table 3. Neonatal outcome at different site clusters

\begin{tabular}{|c|c|c|c|c|c|c|c|c|c|}
\hline & \multirow{2}{*}{$\begin{array}{l}\text { Urban USA } \\
(n=55) \\
\text { mean } \pm S D\end{array}$} & \multirow{2}{*}{$\begin{array}{l}\text { Vienna } \\
(\mathrm{n}=37) \\
\text { mean } \pm \mathrm{SD}\end{array}$} & \multirow{2}{*}{$\begin{array}{l}\text { Rural USA } \\
(\mathrm{n}=39) \\
\text { mean } \pm \mathrm{SD}\end{array}$} & \multicolumn{6}{|c|}{ Significance } \\
\hline & & & & $\mathrm{p}_{\text {site }}$ & $\mathrm{p}_{\mathrm{SD}}$ & $\mathrm{p}_{\mathrm{IA}}$ & $\mathrm{p}_{\mathrm{PH} 1}$ & $\mathrm{p}_{\mathrm{PH} 2}$ & $\mathrm{p}_{\mathrm{PH} 3}$ \\
\hline \multicolumn{10}{|l|}{ Apgar $1 \mathrm{~min}$} \\
\hline Methadone & $7.77 \pm 1.59$ & $8.74 \pm 0.73$ & $7.63 \pm 1.64$ & \multirow[t]{2}{*}{0.001} & \multirow[b]{2}{*}{0.849} & \multirow[b]{2}{*}{0.961} & \multirow[t]{2}{*}{0.004} & \multirow[t]{2}{*}{1.000} & \multirow[t]{2}{*}{0.001} \\
\hline Buprenorphine & $7.80 \pm 1.54$ & $8.89 \pm 0.32$ & $7.60 \pm 1.96$ & & & & & & \\
\hline Buprenorphine & $8.75 \pm 0.55$ & $9.89 \pm 0.47$ & $8.50 \pm 1.47$ & $<0.001$ & 0.691 & 0.828 & $<0.001$ & 1.000 & $<0.001$ \\
\hline \multicolumn{10}{|l|}{ Birth weight, $g$} \\
\hline Methadone & $2,844 \pm 593$ & $2,809 \pm 511$ & $3,017 \pm 490$ & \multirow[t]{2}{*}{0.345} & & & & & \\
\hline Buprenorphine & $3,173 \pm 563$ & $2,972 \pm 494$ & $3,133 \pm 622$ & & 0.044 & 0.629 & & & \\
\hline \multicolumn{10}{|l|}{ Length, $\mathrm{cm}$} \\
\hline Methadone & $47.68 \pm 3.25$ & $48.68 \pm 2.75$ & $48.83 \pm 3.62$ & 0.756 & & & & & \\
\hline \multicolumn{10}{|c|}{ Head circumference, $\mathrm{cm}$} \\
\hline
\end{tabular}

$\mathrm{p}_{\text {site }}=$ Probability site cluster; $\mathrm{p}_{\mathrm{SD}}=$ probability study medication; $\mathrm{p}_{\mathrm{IA}}=$ probability interaction site cluster $\times$ study medication; $\mathrm{p}_{\mathrm{PH} 1}=$ probability post-hoc-1 urban USA vs. Vienna; $\mathrm{p}_{\mathrm{PH} 2}=$ probability post-hoc-2 urban USA vs. rural USA; $\mathrm{p}_{\mathrm{PH} 3}=$ probability posthoc-3 Vienna vs. rural USA.

domized women dropped out soon after transfer to the study medication) occurred at one US urban study center (Johns Hopkins Medical Center), while study medication exposure (methadone vs. buprenorphine) in the three US urban sites together remained balanced even after excluding dropouts with no significant differences between the study medications $(\mathrm{p}=0.293)$.

\section{Neonatal Characteristics}

Regarding Apgar scores, neonates from Vienna were rated significantly higher, irrespective of study drug at 1 and 5 min after birth ( $p=0.001$ and $p<0.001$, respectively). No significant differences between sites were revealed for birth weight, length or head circumference of neonates, but significantly better results for buprenorphine-exposed children in all three outcome parameters (all ps $<0.05$; table 3 ).

\section{Birth Outcome Measurements}

Thirty-seven neonates were born at the Vienna site, 55 in US urban and 39 in US rural sites, with 17 (46\%), 25 $(45 \%)$ and $24(62 \%)$, respectively, being male $(\mathrm{p}=0.251)$. Comparing the three site clusters, no significant difference was observed with regard to preterm rate (methadone: $p=0.364$, buprenorphine: $p=0.794$ ), with 18 of 131 neonates $(13.7 \%)$ born preterm (prior to 37 weeks of preg- nancy), whereof 14 children (78\%) were prenatally exposed to methadone $(\mathrm{p}=0.043)$. The majority of neonates were born vaginally, with a significantly higher cesarean rate in Central Europe/Vienna $(\mathrm{p}=0.024)$.

\section{Results Related to the Neonatal Abstinence Syndrome}

Sixty-eight neonates (52\%) showed signs of NAS requiring treatment, $57 \%$ after methadone exposure and $47 \%$ with intrauterine buprenorphine exposure $(p=0.279)$. Significant site cluster differences were found for NAS treatment parameters, with the US sites reporting more favorable treatment outcome for buprenorphine-exposed neonates, i.e. significantly lower mean treatment durations of 2.5 days at the US rural sites compared to 7 days in Vienna, where no significant inter-medication group differences could be shown (table 4). The higher mean treatment requirement (in days and dosing) after intrauterine methadone exposure is significantly longer in the US urban group with 18 days compared to the US rural group, which had the (significantly) shortest treatment duration requirements independent of opioid medication exposure. No significant correlations between maternal opioid maintenance dose at delivery and NAS scores were revealed. The factor 'site' had no influence regarding this result.

The US rural sites reported significantly shorter mean neonatal hospitalization durations (range 4-79 days, 
Table 4. Site cluster differences in NAS and neonatal outcome variables

\begin{tabular}{|c|c|c|c|c|c|c|c|c|c|}
\hline & \multirow{2}{*}{$\begin{array}{l}\text { Urban USA } \\
(n=55) \\
\text { mean } \pm S D\end{array}$} & \multirow{2}{*}{$\begin{array}{l}\text { Vienna } \\
(\mathrm{n}=37) \\
\text { mean } \pm \mathrm{SD}\end{array}$} & \multirow{2}{*}{$\begin{array}{l}\text { Rural USA } \\
(\mathrm{n}=39) \\
\text { mean } \pm \mathrm{SD}\end{array}$} & \multicolumn{6}{|c|}{ Significance } \\
\hline & & & & $\mathrm{p}_{\text {site }}$ & $\mathrm{p}_{\mathrm{SD}}$ & pIA & PPH1 & $\mathrm{p}_{\mathrm{PH} 2}$ & $\mathrm{p}_{\mathrm{PH} 3}$ \\
\hline \multicolumn{10}{|l|}{ Time of delivery } \\
\hline Meth: full-term & $26(74 \%)$ & $16(84 \%)$ & $17(89 \%)$ & \multirow[t]{2}{*}{$0.364^{\mathrm{a}}$} & & & & & \\
\hline preterm & $9(26 \%)$ & $3(16 \%)$ & $2(11 \%)$ & & & & & & \\
\hline $\begin{array}{l}\text { Bup: full-term } \\
\text { preterm }\end{array}$ & $18(90 \%)$ & $17(94 \%)$ & $19(95 \%)$ & $0.794^{\mathrm{a}}$ & & & & & \\
\hline \multicolumn{10}{|l|}{ Mode of delivery } \\
\hline Meth: vaginal & $26(74 \%)$ & $8(42 \%)$ & $12(63 \%)$ & \multirow[t]{2}{*}{$0.065^{\mathrm{a}}$} & & & & & \\
\hline cesarean & $9(26 \%)$ & $11(58 \%)$ & $7(37 \%)$ & & & & & & \\
\hline \multirow{2}{*}{$\begin{array}{ll}\text { Bup: vaginal } \\
\text { cesarean }\end{array}$} & $16(80 \%)$ & $11(61 \%)$ & $14(70 \%)$ & \multirow[t]{2}{*}{$0.441^{\mathrm{a}}$} & & & & & \\
\hline & $4(20 \%)$ & $7(39 \%)$ & $6(30 \%)$ & & & & & & \\
\hline \multicolumn{10}{|c|}{ Pharmacological treatment for NAS } \\
\hline \multicolumn{10}{|c|}{ Peak MOTHER NAS score } \\
\hline Meth & $13.46 \pm 6.22$ & $12.42 \pm 4.29$ & $11.89 \pm 5.04$ & \multirow[t]{2}{*}{$0.189^{b}$} & & & & & \\
\hline Bup & $10.10 \pm 3.51$ & $13.00 \pm 2.33$ & $10.11 \pm 3.60$ & & $0.026^{\mathrm{b}}$ & & & & \\
\hline \multicolumn{10}{|c|}{ Morphine dose total, mg } \\
\hline Meth & $34.17 \pm 70.72$ & $5.04 \pm 6.71$ & $4.93 \pm 10.56$ & \multirow[t]{2}{*}{$0.017^{\mathrm{b}}$} & & & 0.563 & 0.031 & 0.004 \\
\hline Bup & $1.98 \pm 4.13$ & $1.99 \pm 1.70$ & $1.00 \pm 2.53$ & & $0.021^{\mathrm{b}}$ & & & & \\
\hline \multicolumn{10}{|c|}{ Morphine dose/day, mg } \\
\hline Meth & $0.74 \pm 1.06$ & $0.29 \pm 0.30$ & $0.33 \pm 0.58$ & \multirow[t]{2}{*}{$0.034^{\mathrm{b}}$} & & & 0.666 & 0.048 & 0.008 \\
\hline Bup & $0.13 \pm 0.22$ & $0.21 \pm 0.13$ & $0.08 \pm 0.16$ & & $0.018^{\mathrm{b}}$ & & & & \\
\hline \multicolumn{10}{|l|}{ NAS treatment, days } \\
\hline Meth & $17.91 \pm 22.09$ & $9.26 \pm 8.79$ & $4.92 \pm 6.82$ & $0.006^{\mathrm{b}}$ & & & 0.543 & 0.020 & 0.001 \\
\hline
\end{tabular}

$\mathrm{p}_{\text {site }}=$ Probability site cluster; $\mathrm{p}_{\mathrm{SD}}=$ probability study medication; $\mathrm{p}_{\mathrm{IA}}=$ probability interaction site cluster $\times$ study medication; $\mathrm{p}_{\mathrm{PH} 1}=$ probability post-hoc-1 urban USA vs. Vienna; $\mathrm{p}_{\mathrm{PH} 2}=$ probability post-hoc-2 urban USA vs. rural USA; p $_{\mathrm{PH} 3}=$ probability posthoc-3 Vienna vs. rural USA.

${ }^{a}$ Pearson's $\chi^{2}$ test. ${ }^{b}$ Non-parametric tests (Kruskal-Wallis and Mann-Whitney tests).

mean $10.87, \mathrm{SD}=13.08$ ) compared to Vienna (range 9-31 days, mean $14.76, S D=6.06)$ and the urban US sites (range 2-77 days, mean 17.31, $\mathrm{SD}=18.03)(\mathrm{p}=0.002)$.

\section{Discussion}

A previous publication on screening data of the MOTHER trial already reported significant site cluster differences between patients from urban/rural USA and Central Europe (Vienna), concluding that urban US women might be a particularly vulnerable group with the highest addiction severity of the three investigated groups [17]. The present study showed as well that such site-cluster-specific factors are very important to be able to adequately evaluate study results and to ensure the clinical relevance of the investigation.

This heterogeneity of site clusters remained partly statistically significant in this secondary outcome analysis focusing also on additional variables not having been corrected for in the primary outcome paper [9].

Regarding baseline data at screening and study completion/retention, considerably more similarities have been found between the Central European site and the US 
rural sites, with especially low dropout rates (10 and 17\%, respectively) over a mean treatment duration of 21 weeks until delivery, compared to a discontinuation rate of more than one third of the enrolled US urban inner city study population. This difference might be attributable to the higher prevalence of polysubstance abuse at the urban US sites, since polydrug use among pregnant opioid-dependent patients is associated with premature treatment termination [15]. A variety of research findings support the easier availability of illicit substances in urban areas, particularly the availability of cocaine is known to be increased [20-22]. These differences in cocaine availability have also been shown for European city comparisons, and were confirmed in a multi-site investigation of cocaine consumption patterns in capital cities of the EU, with a significantly lower prevalence for Vienna, which was not comparable to larger US cities [23].

In contrast to cocaine availability, the significantly higher number of THC-positive urine toxicology results at rural US sites is in line with other publications showing a higher availability of cannabis and marijuana in rural areas $[24,25]$. The low percentage of concomitant benzodiazepine consumption during study participation until time of delivery in all three site groups is attributable to benzodiazepine abuse being an exclusion criterion for enrolment in the MOTHER study [9].

The generally low concomitant consumption over the course of pregnancy in this study compared to other RCTs in opioid dependence might be related to the stringent inclusion/exclusion criteria, the intense comprehensive care in a multi-professional setting, including daily visits for medication intake, and the voucher incentives for drug-free urine samples $[14,26]$. Accordingly, the Vienna and the rural US sites showed lower rates of concomitant consumption than the urban US sites and thus earned significantly higher voucher monetary incentives with more than USD 20 difference per week, a finding that supports the positive effect of contingency management in that setting $[27,28]$.

In contrast to the Viennese study population, a large part of screening in randomized US patients was conducted by telephone interviews and medical chart reviews. The personal screening at the Vienna site via faceto-face interviews might have fostered the development of a relationship between patients and caregivers from the beginning on and thus enhanced the women's compliance, suggesting that retention might be particularly influenced by the character of the first contact [29]. Particularly an unfavorable or unsatisfying initiation of relationship can have a negative influence on treatment motivation and retention, especially in psychiatric patients with substance dependence [30].

In addition to inter-site cluster differences in screening procedures affecting relationships between caregivers and patients, and in addiction severity, higher dropout rates in urban US sites might also refer to the medication/medication dissatisfaction with buprenorphine (4 patients medicated with buprenorphine dropped out in Vienna, 4 in rural US sites, and 20 in urban US sites). Transfer onto buprenorphine can be a particular challenge due to the mixed partial agonistic/antagonistic profile, whereas a more homogeneous profile in patients and treatment facility can be beneficial for stabilization. Even if the mean buprenorphine dosing at time of dropout with $14 \mathrm{mg}$ does not suggest underdosing, the early mean dropout time with 9 days after medication induction requires some further investigation [9]. However, these results support the need for opioid medication diversification for pregnant patients.

The mode of delivery does not seem to be a reliable outcome variable, for example the cesarean section rate was higher in Vienna compared to the US site clusters due to different healthcare procedures; whereas cesarean sections are also conducted in Vienna on request, the patients in the US were not offered this option, and cesarean sections were done upon medical indication only.

The beneficial outcome in neonates in favor of buprenorphine is in line with prior publications indicating a significantly longer NAS treatment duration with subsequently higher morphine doses in methadone-exposed infants, independent of applied study design [31, 32]. Nevertheless, various prospective RCTs comparing buprenorphine and methadone at the Vienna Addiction Research Center, including the pioneering study administering buprenorphine during pregnancy [33-37], suggested a milder course of NAS after intrauterine buprenorphine exposure, although not always yielding significant differences from methadone $[5,14,38]$.

The site cluster differences in neonatal outcome might also be related to the different implementation of the study protocol; whereas the minimum length of hospital stay for mothers and neonates in Vienna was 9 days, the minimum hospitalization periods of neonates in urban and rural US sites were only 2 and 4 days, respectively. At US sites, mothers and their newborns were discharged to residential settings where NAS observation and if needed, adaptation of NAS treatment, took place. The standardized tight observation interval at the Vienna site after frequent NAS scoring (intervals of $4 \mathrm{~h}$ ), in contrast to twicedaily scoring with intervals of $8 \mathrm{~h}$ with subsequently dif- 
ferent intervals for morphine applications in especially the urban US centers, may even have led to overrating of symptoms in Vienna over the time course compared to the urban US sites. However, the mean total morphine dose for NAS treatment was still significantly lower at Vienna compared to the urban US sites, where particularly the mean total morphine dose for methadone-exposed newborns was considerably higher in addition to the high standard deviation, suggesting a heterogeneous newborn sample with regard to NAS. When comparing the low standard deviations in Vienna to those of the US sites, the stringent application of the protocol might have resulted in a more homogeneous picture.

The significantly shorter duration of hospitalization at the rural US sites (mean treatment duration of 4 days) could also have been influenced by the significantly higher intrauterine THC exposure - however, there is no evidence for that hypothesis available so far [39, 40]. Moreover, length of hospital stay does not seem to be an adequate treatment outcome indicator since psychosocial reasons frequently lead to longer hospitalizations than medically necessary. The need for treatment requirement is a much more objective outcome indicator that should be considered, particularly in RCTs. Reasons for the different implementation of study procedures might partly be explained by the differences between US and European healthcare systems, which play a particular role in this special patient population. In the US, research participation is frequently the only possibility of being enrolled in treatment, especially in a population of substance-dependent individuals, in contrast to Europe where general practitioners can maintain opioid addicts on methadone or buprenorphine on a lowthreshold basis; moreover, inpatient hospitalization can easily be extended, also on the patient's request [41-44]. Furthermore, to meet Good Clinical Practice requirements, principal investigators in clinical trials in Europe need to be medical doctors, whereas this is no requirement in the US, and the protocol implementation is in the principal investigator's responsibility. Additionally, medical insurance coverage for study participants is mandatory in Europe, entailing that the principal investigators stick as strictly as possibly to the submitted protocol to ensure the insurance coverage. Thus, regarding neonatal treatment procedures, the children in Vienna had to stay in hospital for at least 9 days, with a roomingin option for the mother in contrast to the newborns at the US sites.

Finally, it has to be highlighted that not only medication aspects, but also environmental as well as cultural aspects or regional disparities of study participants should be considered in multi-site research planning, study implementation and interpretation of results.

\section{Conclusion}

Data collected in the MOTHER trial have enabled a unique comparison between Central European/Viennese and rural/urban US pregnant opioid-dependent women and have shown differences in pretreatment characteristics, addiction severity and pregnancy outcomes [9]. Site cluster differences in many relevant sociodemographic and clinical variables as well as in healthcare systems might influence therapeutic interventions to a great extent and, thus, need to be considered from the beginning on when planning a study. As the first international study on opioid-dependent pregnant women living on different continents, it represents a role model for pharmacological treatment during pregnancy and provides valuable lessons for future research on opioid-dependent women and outcome of their neonates. Moreover, study procedures that have turned out to be beneficial at different site clusters should be transferred into clinical practice.

\section{Acknowledgements}

We would like to thank the nursing staff of the Vienna Addiction Clinic, the Department for Gynecology and Obstetrics and the Department for Neonatology. Moreover, we would like to thank the principal investigators of our multi-site research team: Hendree Jones (Johns Hopkins University), Karol Kaltenbach (Thomas Jefferson University), Sarah Heil (University of Vermont), Mara Coyle (Brown University), Peter Martin (Vanderbilt University) and Susan Stine (Wayne State University). This research was supported by the following grants from the National Institute on Drug Abuse: R01DA015778 (Brown University), R01DA15832 (Wayne State University), R01DA015764 (Johns Hopkins University), R01DA015738 (Thomas Jefferson University), R01DA015741 (University of Toronto), R01DA017513 and M01RR00095 (General Clinical Research Center, Vanderbilt University), R01DA018410 (University of Vermont) and R01DA018417 (Medical University of Vienna).

\section{Disclosure Statement}

Andjela Baewert received financial support for presentations from Reckitt Benckiser and Schering Plough.

This research was supported by NIDA: grant number R01-DA018417. IRB number, Medical University of Vienna: IRB $451 / 1998$. 


\section{References}

1 UNODC: World Drug Report 2010. United Nations, New York, 2010. Available at http:// w w w. unodc.org/documents/wdr/ WDR_2010/World_Drug_Report_2010_ lo-res.pdf (accessed 28.9.2011).

-2 Behnke M, Eyler FD, Conlon M, Casanova OQ, Woods NS: How fetal cocaine exposure increases neonatal hospital costs. Pediatrics 1997;99:204-208.

>3 Derauf C, Kekatpure M, Neyzi N, Lester B, Kosofsky B: Neuroimaging of children following prenatal drug exposure. Semin Cell Dev Biol 2009;20:441-454.

4 Cavanaugh CE, Latimer WW: Recent sex trade and injection drug use among pregnant opiate- and cocaine-dependent women in treatment: the significance of psychiatric comorbidity. Addict Disord Their Treat 2010;9:32-40.

$>5$ Fischer G: Treatment of opioid dependence in pregnant women. Addiction 2000;95: 1141-1144.

6 Winklbaur B, Baewert A, Jagsch R, Rohrmeister K, Metz V, Aeschbach Jachmann C, Thau K, Fischer G: Association between prenatal tobacco exposure and outcome of neonates born to opioid-maintained mothers. Implications for treatment. Eur Addict Res 2009;15:150-156.

$>7$ Lauder W, Roxburgh M, Harris J, Law J: Developing self-neglect theory: analysis of related and atypical cases of people identified as self-neglecting. J Psychiatr Ment Health Nurs 2009;16:447-454.

$>8$ Grella CE, Karno MP, Warda US, Niv N, Moore AA: Gender and comorbidity among individuals with opioid use disorders in the NESARC study. Addict Behav 2009;34:498504.

$>9$ Jones HE, Kaltenbach K, Heil SH, Stine SM, Coyle MG, Arria AM, O'Grady KE, Selby P, Martin PR, Fischer G: Neonatal abstinence syndrome following methadone or buprenorphine exposure. N Engl J Med 2010; 363:2320-2331.

$>10$ Winklbaur B, Kopf N, Ebner N, Jung E, Thau $\mathrm{K}$, Fischer G: Treating pregnant women dependent on opioids is not the same as treating pregnancy and opioid dependence: a knowledge synthesis for better treatment for women and neonates. Addiction 2008;103: 1429-1440.

$>11$ Fiellin DA, Kleber H, Trumble-Hejduk JG, McLellan AT, Kosten TR: Consensus statement on office-based treatment of opioid dependence using buprenorphine. J Subst Abuse Treat 2004;27:153-159.

$>12$ Unger A, Jung E, Winklbaur B, Fischer G: Gender issues in the pharmacotherapy of opioid-addicted women: buprenorphine. J Addict Dis 2010;29:217-230.
13 Jones HE, Johnson RE, Jasinski DR, O'Grady KE, Chisholm CA, Choo RE, Crocetti M, Dudas R, Harrow C, Huestis MA, Jansson LM, Lantz M, Lester BM, Milio L: Buprenorphine versus methadone in the treatment of pregnant opioid-dependent patients: effects on the neonatal abstinence syndrome. Drug Alcohol Depend 2005;79:1-10.

14 Fischer G, Ortner R, Rohrmeister K, Jagsch $\mathrm{R}$, Baewert A, Langer M, Aschauer H: Methadone versus buprenorphine in pregnant addicts: a double-blind, double-dummy comparison study. Addiction 2006;101:275-281.

15 Stine SM, Heil SH, Kaltenbach K, Martin PR, Coyle MG, Fischer G, Arria AM, Selby P, Jones HE: Characteristics of opioid-using pregnant women who accept or refuse participation in a clinical trial: screening results from the MOTHER study. Am J Drug Alcohol Abuse 2009;35:429-433.

16 Jones HE, Martin PR, Heil SH, Kaltenbach K, Selby P, Coyle MG, Stine SM, O'Grady KE, Arria AM, Fischer G: Treatment of opioiddependent pregnant women: clinical and research issues. J Subst Abuse Treat 2008;35: 245-259.

17 Unger AS, Martin PR, Kaltenbach K, Stine SM, Heil SH, Jones HE, Arria AM, Coyle MG, Selby P, Fischer G: Clinical characteristics of Central European and North American samples of pregnant women screened for opioid agonist treatment. Eur Addict Res 2010;16:99-107.

- 18 Finnegan LP, Connaughton JF Jr, Kron RE, Emich JP: Neonatal Abstinence Syndrome: assessment and management. Addict Dis 1975;2:141-158.

-19 McLellan AT, Kushner H, Metzger D, Peters R, Smith I, Grissom G, Pettinati H, Argeriou M: The Fifth Edition of the Addiction Severity Index. J Subst Abuse Treat 1992;9:199213.

20 Martino SC, Ellickson PL, McCaffrey DF: Developmental trajectories of substance use from early to late adolescence: a comparison of rural and urban youth. J Stud Alcohol Drugs 2008;69:430-440.

21 Metsch LR, McCoy CB: Drug treatment experiences: rural and urban comparisons. Subst Use Misuse 1999;34:763-784.

22 Heil SH, Sigmon SC, Jones HE, Wagner M: Comparison of characteristics of opioid-using pregnant women in rural and urban settings. Am J Drug Alcohol Abuse 2008;34: 463-471.

23 Bäwert A, Primus N, Jagsch R, Eder H, Zanki M, Thau K, Fischer G: Cocaine abuse in Vienna and European cities - a multi-centre study. Wien Klin Wochenschr 2006;118:521530.

$>24$ Dunn MS, Ilapogu V, Taylor L, Naney C, Blackwell R, Wilder R, Givens C: Self-reported substance use and sexual behaviors among adolescents in a rural state. J Sch Health 2008;78:587-593.
25 Rostosky SS, Owens GP, Zimmermann RS, Riggle ED: Associations among sexual attraction status, school belonging, and alcohol and marijuana use in rural high school students. J Adolesc 2003;26:741-751.

26 Morgenstern J, Blanchard KA, Kahler C, Barbosa KM, McCrady BS, McVeigh KH: Testing mechanisms of action for intensive case management. Addiction 2008;103:469477.

27 Brooner RK, Kidorf MS, Stoller KB, Neufeld KJ, Kolodner K: Comparing adaptive stepped care and monetary-based voucher intervention for opioid dependence. Drug Alcohol Depend 2007;88(suppl 2):14-23.

28 Henderson M, Wight D, Nixon C, Hart G: Retaining young people in a longitudinal sexual health survey: a trial of strategies to maintain participation. BMC Med Res Methodol 2010;10:9.

29 Meyers K, Webb A, Frantz J, Randall M: What does it take to retain substance-abusing adolescents in research protocols? Delineation of effort required, strategies undertaken, costs incurred, and 6-month posttreatment differences by retention difficulty. Drug Alcohol Depend 2003;69:73-85.

30 Kelly SM, O’Grady KE, Brown BS, Mitchell SG, Schwartz RP: The role of patient satisfaction in methadone treatment. Am J Drug Alcohol Abuse 2010;36:150-154.

31 Colombini N, Elias R, Busuttil M, Dubuc M, Einaudi MA, Bues-Charbit M: Hospital morphine preparation for abstinence syndrome in newborns exposed to buprenorphine or methadone. Pharm World Sci 2008; 30:227-234.

32 Binder T, Vavrinková B: Prospective randomised comparative study of the effect of buprenorphine, methadone and heroin on the course of pregnancy, birth weight of newborns, early postpartum adaptation and course of the neonatal abstinence syndrome in women followed up in the outpatient department. Neuro Endocrinol Lett 2008;29:80-86.

33 Eder H, Fischer G, Gombas W, Jagsch R, Stühlinger G, Kasper S: Comparison of buprenorphine and methadone maintenance in opiate addicts. Eur Addict Res 1998;4 (suppl 1):3-7.

34 Fischer G, Johnson RE, Eder H, Jagsch R, Peternell A, Weninger M, Langer M, Aschauer HN: Treatment of opioid-dependent pregnant women with buprenorphine. Addiction 2000;95:239-244.

35 Schindler SD, Eder H, Ortner R, Rohrmeister K, Langer M, Fischer G: Neonatal outcome following buprenorphine maintenance during conception and throughout pregnancy. Neonatal outcome following buprenorphine maintenance during conception and throughout pregnancy. Addiction 2003;98: 103-110. 
36 Ortner R, Jagsch R, Schindler SD, Primorac A, Fischer G, General Practitioner Addiction Team. Buprenorphine maintenance: officebased treatment with addiction clinic support. Eur Addict Res 2004;10:105-111.

37 Fischer G, Ortner R, Rohrmeister K, Jagsch $\mathrm{R}$, Baewert A, Langer M, Aschauer H: Methadone versus buprenorphine in pregnant addicts: a double-blind, double-dummy comparison study. Addiction 2006;101:275-281.

38 Unger A, Jagsch R, Jones H, Arria A, Leitich $\mathrm{H}$, Rohrmeister K, Aschauer C, Winklbaur B, Bäwert A, Fischer G: Randomized controlled trials in pregnancy: scientific and ethical aspects exposure to different opioid medications during pregnancy in an intraindividual comparison. Addiction 2011;106: 1355-1362.
39 Fride E: The endocannabinoid-CB(1) receptor system in pre- and postnatal life. Eur J Pharmacol 2004;500:289-297.

40 Stone KC, LaGasse LL, Lester BM, Shankaran S, Bada HS, Bauer CR, Hammond JA: Sleep problems in children with prenatal substance exposure: the Maternal Lifestyle study. Arch Pediatr Adolesc Med 2010;164: 452-456.

41 Wilkin D: Restructuring primary and community health services in four countries: from cottage industry to integrated provider? Health Soc Care Community 2002;10: 309-312.
42 Wilensky HL: Social science and the public agenda: reflections on the relation of knowledge to policy in the United States and abroad. J Health Polit Policy Law 1997;22: 1241-1265.

43 Aeschbach Jachmann C, Jagsch R, Winklbaur B, Matzenauer C, Fischer G: Officebased treatment in opioid dependence: a critical survey of prescription practices for opioid maintenance medications and concomitant benzodiazepines in Vienna, Austria. Eur Addict Res 2008;14:206-212.

44 Keen J, Oliver P, Rowse G, Mathers N: Does methadone maintenance treatment based on the new national guidelines work in a primary care setting? Br J Gen Pract 2003;53: 461-467. 